\title{
Synthesis, Structural Characterization and Multifrequency Electron Paramagnetic Resonance Studies of Mononuclear Thiomolybdenyl Complexes
}

Simon C. Drew, ${ }^{a, b}$ Jason P. Hill, ${ }^{c}$ Ian Lane, ${ }^{a, b}$ Graeme R. Hanson, ${ }^{*}, a, b$ Robert W. Gable ${ }^{c}$ and Charles G. Young ${ }^{*}, \mathrm{c}$

${ }^{\mathrm{a}}$ Centre for Magnetic Resonance and ${ }^{\mathrm{b}}$ Centre for Metals in Biology, University of Queensland, 4072, Australia, ${ }^{\mathrm{c}}$ School of Chemistry, University of Melbourne, Victoria 3010, Australia

X-ray crystallographic files for $\mathrm{Tp} * \operatorname{MoS}($ cat $), \mathrm{Tp} * \mathrm{MoS}(\mathrm{pp})_{2}$ and $\mathrm{Tp} * \mathrm{MoO}(\mathrm{etp})_{2}$ in CIF format. Table S1, isotropic spin Hamiltonian parameters for $\mathrm{Mo}(\mathrm{V})$ model complexes determined from room temperature S- and X-band EPR spectra.

Figure S1, a comparison of simulated EPR spectra for $\mathrm{Tp} * \mathrm{MoO}(\mathrm{bdt})$ in orthorhombic and monoclinic $\left(\mathrm{C}_{\mathrm{s}}\right)$ symmetries.

Figure S2, a comparison of simulated EPR spectra for $\mathrm{Tp} * \mathrm{MoO}(\mathrm{cat})$ in orthorhombic and monoclinic $\left(\mathrm{C}_{\mathrm{s}}\right)$ symmetries. 
Table S1: Isotropic spin Hamiltonian parameters for $\mathrm{Mo}(\mathrm{V})$ model complexes determined from room temperature S- and X-band EPR spectra.

\begin{tabular}{|c|c|c|c|c|}
\hline \multirow[t]{2}{*}{ Complex } & \multicolumn{2}{|c|}{ S-Band } & \multicolumn{2}{|c|}{ X-band } \\
\hline & $g_{\text {iso }}^{\dagger}$ & $\mathrm{A}_{\text {iso }}^{\dagger}$ & $\mathrm{g}_{\text {iso }}^{\dagger}$ & $\mathrm{A}_{\text {iso }}^{\dagger}$ \\
\hline $\mathrm{Tp} * \operatorname{MoS}(\mathrm{etp})_{2}$ & 1.9057 & 46.5 & - & - \\
\hline $\mathrm{Tp} * \mathrm{MoO}(\mathrm{etp})_{2}$ & 1.9335 & 47.2 & 1.9341 & 46.6 \\
\hline $\mathrm{Tp}^{*} \operatorname{MoS}(\mathrm{pp})_{2}$ & 1.9056 & 47.0 & 1.9171 & 46.5 \\
\hline $\mathrm{Tp}^{*} \mathrm{MoO}(\mathrm{pp})_{2}$ & 1.9375 & 45.6 & 1.9387 & 45.0 \\
\hline $\mathrm{Tp} * \mathrm{MoS}(\mathrm{OPh})_{2}$ & 1.9044 & 47.0 & 1.9041 & 47.0 \\
\hline $\mathrm{Tp}^{*} \mathrm{MoO}(\mathrm{OPh})_{2}^{\mathrm{a}}$ & - & - & 1.932 & 44.2 \\
\hline Tp*MoS(cat) & 1.9466 & 41.9 & 1.9364 & 41.5 \\
\hline $\mathrm{Tp} * \mathrm{MoO}$ (cat) & 1.9497 & 38.9 & 1.9497 & 38.9 \\
\hline $\mathrm{Tp}^{*} \mathrm{MoO}(\mathrm{cat})^{\mathrm{b}}$ & - & - & 1.953 & 40.1 \\
\hline Tp*MoS(bdt) & 1.9662 & 37.0 & 1.9661 & 36.5 \\
\hline $\mathrm{Tp} * \mathrm{MoO}(\mathrm{bdt})$ & 1.97095 & 34.9 & 1.97095 & 34.9 \\
\hline $\mathrm{Tp}^{*} \mathrm{MoO}(\mathrm{bdt})^{\mathrm{c}}$ & - & - & 1.971 & 37.0 \\
\hline Tp*MoS(tdt) & 1.9616 & 36.8 & 1.9617 & 37.0 \\
\hline
\end{tabular}

${ }^{\dagger}$ Units for hyperfine coupling constants $=10^{-4} \mathrm{~cm}^{-1}$

${ }^{a}$ Reference [1].

${ }^{\mathrm{b}}$ Reference [2]. Basu, P.; Bruck, M.A.; Li, Z.; Dhawan, I.K.; Enemark, J.H. Inorg. Chem., 1995, 34, 405-407.

${ }^{\mathrm{c}}$ Reference [3]. Dhawan, I.K.; Enemark, J.H. Inorg. Chem., 1996, 35, 4873-4882. 

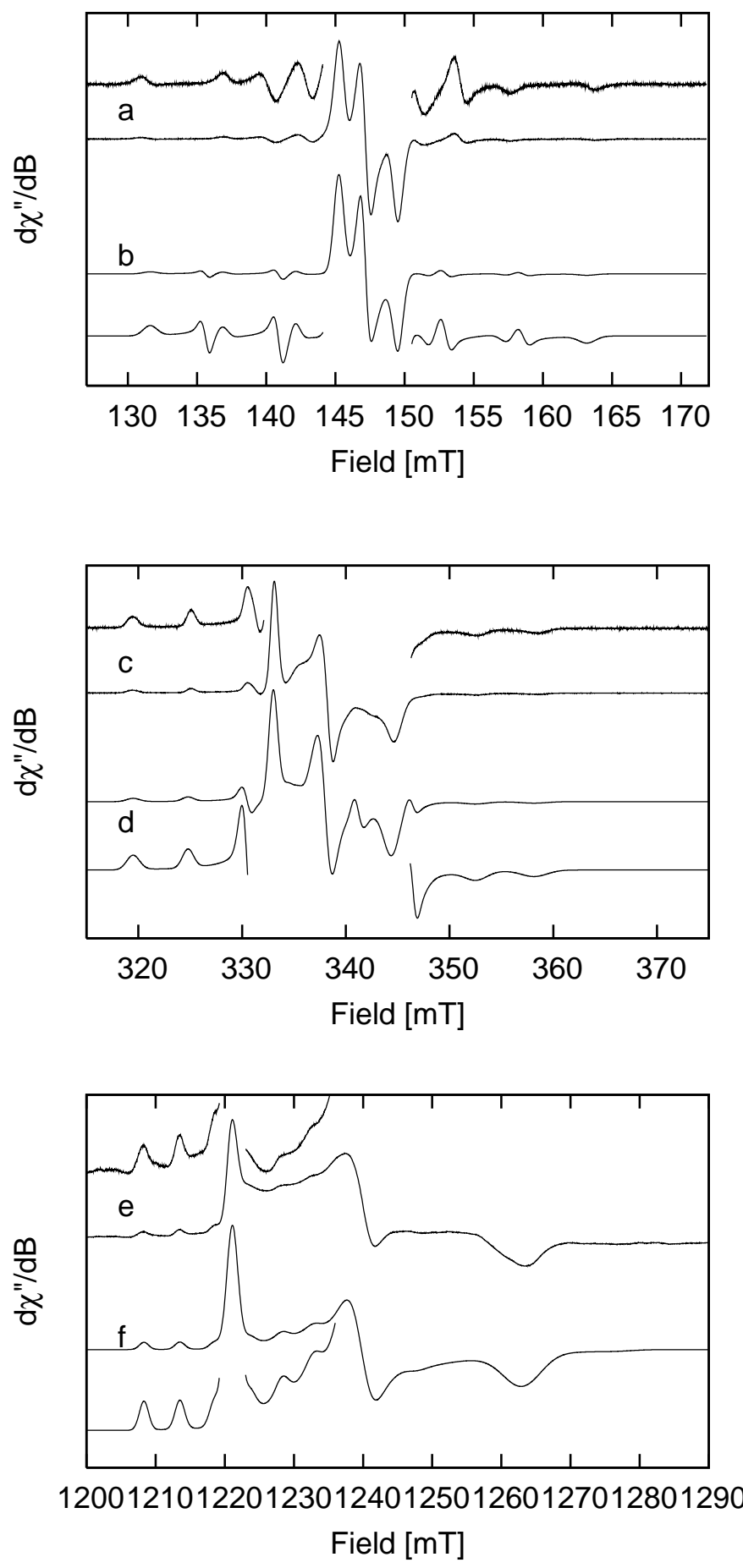

Figure S1. Multifrequency EPR spectra of $\mathrm{Tp} * \mathrm{MoO}(\mathrm{bdt})$ at $130 \mathrm{~K}$ and comparison with simulations based upon the high symmetry parameters of Dhawan and Enemark [3]. The linewidth parameters are identical to those used in Figure 8 (main text). (a) S-band experimental spectrum, $v=4.0630$ GHz; (b) simulated S-band spectrum; (c) X-band experimental spectrum, v=9.3338GHz; (d) simulated X-band spectrum; (e) Q-band experimental spectrum, v=34.226GHz; (f) simulated Q- 
band spectrum; $(\mathrm{a}, \mathrm{b})$ Both insets expanded by a factor of 5.0; $(\mathrm{c}, \mathrm{d})$ Both insets expanded by a factor of 4.0; (e,f) Low field insets expanded by 5.0 and 4.0 respectively. 

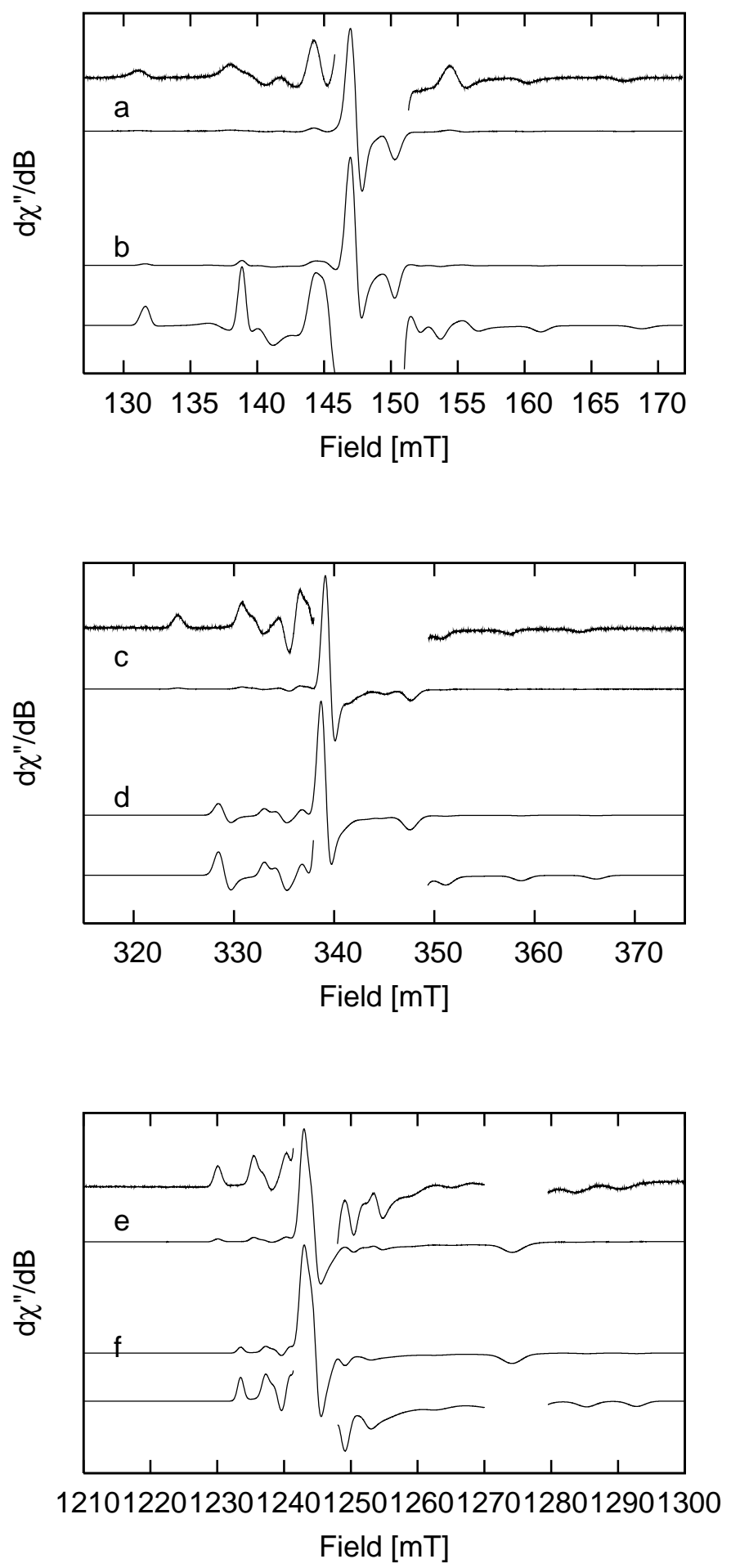

Figure S2. Multifrequency EPR spectra of $\mathrm{Tp} * \mathrm{MoO}$ (cat) at $130 \mathrm{~K}$ and comparison with simulations based upon the high symmetry parameters of Basu et al. [2].The linewidth parameters are identical to those used in Figure 7 (main text). (a) S-band experimental spectrum, v=4.0630 GHz; (b) simulated S-band spectrum; (c) X-band experimental spectrum, $v=9.3338 \mathrm{GHz}$; (d) simulated X-band spectrum; (e) Q-band experimental spectrum, v=34.226GHz; (f) simulated Q-band spectrum; (a,b) Both insets expanded by a factor of 12.0; (c,d) Low-field insets expanded by a factor of 12.0 and 
2.0, respectively and high-field insets expanded by factor of 12.0 and 10.0, respectively; (e,f) Low field insets expanded by 7.0 and 4.0 respectively and high-field insets expanded by 15.0 and 10.0, respectively.

\section{References}

[1] Cleland, W. E., Jr.; Barnhart, K. M.; Yamanouchi, K.; Collison, D.; Mabbs, F. E.; Ortega, R.B.; Enemark, J. H. Inorg. Chem. 1987, 26, 1017.

[2] Basu, P.; Bruck, M. A.; Zheng, L.; Dhawan, I. K.; Enemark, J. H. Inorg. Chem. 1995, 34, 405.

[3] Dhawan, I. K.; Enemark, J. H. Inorg. Chem. 1996, 35, 4873. 